\title{
Regulatory RNAs derived from transfer RNA?
}

\author{
THORU PEDERSON ${ }^{1,2}$
}

${ }^{1}$ Department of Biochemistry and Molecular Pharmacology, University of Massachusetts Medical School, Worcester, Massachusetts 01605 USA

${ }^{2}$ Program in Cell and Developmental Dynamics, University of Massachusetts Medical School, Worcester, Massachusetts 01605 USA

\begin{abstract}
Four recent studies suggest that cleavages of transfer RNAs generate products with microRNA-like features, with some evidence of function. If their regulatory functions were to be confirmed, these newly revealed RNAs would add to the expanding repertoire of small noncoding RNAs and would also provide new perspectives on the coevolution of transfer RNA and messenger RNA.
\end{abstract}

Keywords: Argonautes; deep sequencing; microRNAs; transfer RNA

\section{DEEP SEQUENCING REVEALS TRANSFER RNA-DERIVED REGULATORY RNAS}

The discovery of microRNAs was a milestone in the modern era of biology (Lee et al. 1993; Wightman et al. 1993). The many hundreds of microRNAs in an organism are processed from gene transcripts that produce both them and messenger RNAs, or from genes that have evolved to produce only microRNAs. Related pathways produce endogenous small interfering RNAs and the germline-expressed piRNAs (Ghildiyal and Zamore 2009). Now, four nearly contemporaneous papers have revealed another class of small noncoding RNAs with microRNA features. They are derived from a pioneer entry in the RNA discovery chain: transfer RNA (Hoagland 2004).

There had been previous reports of transfer RNA-derived fragments (Lee and Collins 2005; Calabrese et al. 2007; Babiarz et al. 2008; Kawaji et al. 2008). But, the new work reviewed here took this to a more refined analytical depth and has included experiments that address the biogenesis and function of transfer RNA-derived microRNA-like RNAs.

First in the current wave of studies was an investigation of small RNAs in HIV-infected cells (Yeung et al. 2009). In addition to detecting several small noncoding RNAs that were known from previous work to be processed from the viral RNA itself, this study revealed the presence in HIVinfected cells of a small RNA corresponding to nucleotides $57-76$ of the host cell tRNA ${ }^{\text {Lys3 }}$ that is known to bind to a site

Reprint requests to: Thoru Pederson, Department of Biochemistry and Molecular Pharmacology, University of Massachusetts Medical School, Worcester, MA 01605 USA; e-mail: thoru.pederson@umassmed.edu; fax: (508) 856-8668.

Article published online ahead of print. Article and publication date are at http://www.rnajournal.org/cgi/doi/10.1261/rna.2266510.
(PBS) in the genomic HIV, where it serves as the primer for reverse transcription. Additional experiments demonstrated that the cellular prevalence of this 20-nucleotide (nt) RNA was correlated with the HIV expression level; that this tRNAderived small RNA was bound to a canonical Argonaute protein, Ago 2; that it could silence a luciferase reporter engineered to be a target; and that a duplex formed by annealing this RNA to the HIV RNA PBS is a substrate for Dicer cleavage in vitro.

Only 2 mo later, Lee et al. (2009) reported a deepsequencing analysis of total small RNAs from two human prostatic carcinoma cell lines. They got $>600,000$ reads that included 635 out of the 695 microRNAs in the Sanger database. Among the remainder they found 17 RNAs, 18$22 \mathrm{nt}$ in length, that aligned with transfer RNA sequences. Five were derived from the $5^{\prime}$ ends of mature tRNAs, eight were derived from the $3^{\prime}$ ends of mature tRNAs, and four were derived from the $3^{\prime}$ trailer regions of pre-tRNAs. The cloning coverage indicated that these tRNA-related small RNAs are more abundant in these cells than the majority of microRNAs, and are within an order of magnitude as abundant as the most prevalent microRNAs. Spirited by these findings, Lee et al. (2009) dove back into the pot of all the other noncanonical microRNAs in their library (i.e., ones not in the Sanger database) and turned up another 621 that correspond to tRNA sequences (this large number obviously reflecting many different fragments from the much smaller number of tRNA species).

To examine biological function, Lee et al. (2009) chose one of their RNAs, tRF-1001, which corresponds to the 3' trailer of pre-tRNA ${ }^{\text {SerTGA }}$. They found that it displayed an elevated expression in various human cell lines relative to adult organs and tissues and that, among the cell lines, its expression was correlated with proliferation rate. They succeeded in 
knocking down tRF-1001 with siRNA (this itself was interesting, as it was a result that could not have been assumed) and found that this slowed cell proliferation. This effect was blocked by the cointroduction of a $2^{\prime}$-O-methyl version of tRF-1001 together with the siRNA. Finally, they reported that the production of tRF-1001 involves ELAC2, a homolog of the pre-tRNA $3^{\prime}$-processing enzyme RNase Z, which is compatible with the fact that tRF-1001 is derived from a tRNA 3' trailer.

In a publication appearing only a few weeks later Cole et al. (2009) reported a deep-sequencing study in HeLa cells that also revealed numerous RNAs corresponding to transfer RNA fragments. Most of their reads corresponded to the 5 ' ends of mature tRNAs. They selected a tRNA ${ }^{\text {Gln }}$ for further study and showed that it is cleaved by Dicer both in vivo and in vitro, although one might have hoped that they would have examined this with respect to more than a single tRNA. They went on to find that this tRNA ${ }^{\text {Gln }}$-derived RNA was more sensitive to knockdown of Dicer than were bona fide microRNAs, although the possibility was not examined that this hypersensitivity might reflect a general destabilization of tRNA due to a slowing of growth resulting from Dicer knockdown. They further found that although their tRNAderived RNAs were bound to Ago 1 and Ago 2 to some degree, this complexing was much less stoichiometric than for canonical microRNAs. Moreover, Cole et al. (2009) found that these tRNA-derived RNAs carried blocked 2'-OH termini. In all of these respects, these tRNA-derived RNAs differ from typical mammalian microRNAs. Thus, although these findings, taken together, were indeed provocative, the picture remained rather unsettled.

Subsequently (4 mo later), Haussecker et al. (2010) reported a study of small noncoding RNAs in the adenovirus-transformed human embryonic kidney 293 cell line, which again revealed transfer RNA-derived species. Owing to the way they got into this from a previous study, they used a combined decapping and RNA ligation strategy. For our purposes, the decapping aspect is irrelevant (as it proved to be in their study) and the sequencing was based on the ability of T4 RNA ligase to covalently circularize RNAs bearing a $5^{\prime}$ phosphate and a $3^{\prime}$ hydroxyl (and to also conjoin two or more such molecules, which is beyond the scope of our discussion here). This assay is based on the resulting electrophoretic gel shift (or disappearance) of certain RNA species. Using this approach they observed two populations of tRNA-derived RNAs. One corresponded to the $3^{\prime}$ ends of mature tRNAs. Their presence was found to be be Dicer dependent. Haussecker et al. (2010) termed these "Type I." The second population of tRNA-derived RNAs corresponded to the $3^{\prime}$ ends of pre-tRNAs, and this group was termed "Type II." Their production was Dicer independent and presumably involves the action of RNase $\mathrm{Z}$ in the standard pre-tRNA 3' processing pathway.

The type I RNAs were found to be complexed with Argonautes 1-4, but not Mov10. In contrast, the type II
RNAs were found to be complexed with Argonautes 3 and 4 and to a much lesser extent to Argonautes 1 and 2, and not at all to Mov 10. Haussecker et al. (2010) further reported that when any one of Argonautes 1-4 was overexpressed, the level of Type II tRNA-derived RNAs increased. But, as silencing experiments were undertaken with standard luciferase reporters, things began to get even more interesting.

\section{A surprise}

When a dual Renilla-Firefly luciferase construct bearing a target for one of the Type I RNAs, "cand14," was transfected into cells expressing this RNA, the addition of an antisense oligo to cand 14 resulted in a modest (30\%-40\%) elevation of reporter expression, consistent with a role of this RNA in silencing. (The investigators speculated that the limited silencing observed might reflect the preferential association of cand14 with Ago 3 and Ago 4, relative to Ago 1 or Ago 2, as well as other possible factors.) In contrast to these results with cand14, when a comparable experiment was performed with cells expressing a Type II RNA, "cand45," and a reporter containing a cand 45 target, the addition of an antisense oligo to cand45 did not result in an elevation of reporter expression, indicating that, unlike cand14, cand45 does not play a role in silencing. The investigators considered various possibilities for this contrasting finding and decided, as one potential approach, to overexpress cand45, reasoning that perhaps its prevalence in these cells simply was inadequate relative to the reporter target levels being expressed. However, this still did not lead to evidence of silencing by cand 45 . In fact, they observed that the addition of the oligo antisense to cand45 actually increased silencing, a phenomenon they termed sense-induced trans-silencing (SITS).

They further found that SITS involved all three cand45 target sites in the reporter and that it was not dependent on the chemistry of the antisense oligo used-a completely substituted ribose-2'-O-methyl oligo was as effective as the initial cand 45 oligo they had used, which contained a trio of unmodified deoxy, ribose-2'-O-methyl and locked nucleic acid nucleotides. Indeed, the fully substituted 2 '-O-methyl antisense oligo was observed to induce reporter silencing even when cand 45 was not overexpressed. Finally, Haussecker et al. (2010) also observed SITS in a mouse embryonic cell line, indicating that this phenomenon is not a quirk of human 293 cells. Though presently unexplained as to mechanism, these provocative findings point to a distinction between the messenger RNA action of certain of these tRNA-derived RNAs versus that of canonical microRNAs, and could possibly be the most important finding Haussecker et al. (2010) made in their study.

\section{A deeper look at deep sequencing}

Current RNA cloning and deep sequencing methods are so sensitive that one expects virtually every RNA fragment 
present in a cell at a few copies (maybe even one) to be captured (unless either end is blocked from taking the adaptor linkers and cannot be enzymatically converted into an unblocked end). So, obviously, the first question is whether these tRNA-related small RNAs are simply a steady-state snapshot of a cell's metabolic turnover of tRNAs. The results argue against this hypothesis. As mentioned, Lee et al. (2009) found that five of the RNAs aligned with the $5^{\prime}$ ends of various tRNAs, eight corresponded to tRNA $3^{\prime}$ ends, and four mapped to the $3^{\prime}$ trailers of pre-tRNAs. Many fewer of the reads corresponded to the anticodon stemloop regions of tRNAs. The degree of nonrandomness was even greater for the RNAs reported by Cole et al. (2009), which predominantly mapped to the $5^{\prime}$ ends of mature tRNAs and extending $3^{\prime}$-ward to just beyond the D-loop. Moreover, these investigators noted a noncorrelation (indeed, an anticorrelation) between the prevalence of reads in the deep sequencing and the known abundance of various tRNAs in HeLa cells, and a comparable noncorrelation with the anticodon prevalence of isoaccepting tRNAs. Moreover, they noted that the most prevalent tRNA-derived RNAs were from three classes of tRNAs whose coding (Gln, Lys, and Val) do not correspond to the prevalence of these amino acids in HeLa cell proteins. In addition, the data of Haussecker et al. (2010) also point to these tRNA-derived RNAs as being nonrandom cleavage products.

\section{Bona fide microRNAs?}

One of course next asks: Are they really microRNAs? We can first ask: What is the definition of a microRNA? If the definition is that of an RNA derived from a pol II transcript by Drosha processing in the nucleus, export of a pre-microRNA and Dicer action, then these tRNA-derived RNAs are disqualified from the outset since they are pol III transcripts. But the evidence for processing of at least some of them by Dicer seems provocative, so by that criterion those may qualify. Another key criterion is Argonaute association. Here the evidence is a bit wobbly among the studies, but there is nevertheless a sense of connectivity. The experimental redirection of one of these RNAs into Ago 2 complexes (Cole et al. 2009) seems provocative as to both function and interactivity with the canonical microRNA pathway. Meanwhile, the finding of blocked $3^{\prime}$ termini on these RNAs, presumed (but not demonstrated to be) ribose- $2^{\prime}$-O-methyl groups (Cole et al. 2009) is a differentiating feature, since this modification has so far not been found in mammalian microRNAs. This suggests that these tRNA-derived RNAs are perhaps siblings, but not identical twins, of bona fide mammalian microRNAs.

The starkest deficiency in all of these four studies was the lack of mRNA target identification for these tRNA-derived RNAs. There can be no meaningful progress until this is done. Target identification is a challenging bioinformatics endeavor but should be no more so for these tRNA-derived
RNAs than for the extant microRNA databases that have been so analyzed to date.

A curious twist is that, as chance would have it, the very "Type II" tRNA-derived RNA chosen for scrutiny by Haussecker et al. (2010) (cand45) is none other than tRF1001, as investigated by Lee et al. (2009). To the extent that comparable experiments were performed in the two studies, the results were concordant (although Lee et al. (2009) did not delve far enough to discover SITS). That said, in today's very fast moving small regulatory RNA field we might not want to jump too quickly into holding up these four manuscripts as necessarily constituting an international tribunal of consensus. The evidence that these transfer RNA-derived RNAs are actually microRNAs presently hinges on the criteria of Argonaute association and the silencing results, the latter less subject to debate than the former. (After all, there may well be new Argonautes and related proteins awaiting discovery.) And why were these tRNA-derived RNAs not seen in other deep-sequencing studies, of which there had been many in the preceding year or so? Perhaps in previous studies the investigators decided to simply not pursue reads that were not in the Sanger microRNA database. Cole et al. (2009) thoughtfully consider other possibilities in their Discussion.

\section{Biogenesis}

On this front (of interest to both the longstanding RNA processing community as well as the more recently expanding small regulatory RNA field), we presently have more questions than answers. How do the cleavages predicted to produce these RNAs navigate around the canonical processing enzymes that would also be engaging these molecules, that is, RNase $\mathrm{P}$ and RNase $\mathrm{Z}$ at the $5^{\prime}$ and $3^{\prime}$ termini of pretRNAs, respectively, and subsequently, the aminoacyl-tRNA synthetases? Where in the cell are these tRNA-derived RNAs produced? $5^{\prime}$ and $3^{\prime}$ processing of tRNA takes place in the nucleus (Hopper et al. 2010), but where do the cleavages needed to produce these observed tRNA-derived regulatory RNAs occur? Dicer cleavage of pre-microRNAs (70-nt long, hauntingly similar to the length of tRNAs, but with one loop versus four in the latter) is thought to be cytoplasmic, but to the extent that some of these newly discovered RNAs are the $3^{\prime}$ trailers of pre-tRNAs, the nucleus is implicated as the site (Hopper et al. 2010). One also wonders whether these tRNAderived small RNAs might compete with other processes in which full-length tRNAs have been implicated, for example, in the splicing of certain pre-mRNAs (Zamboni et al. 2009). It is also of interest to consider how these new findings might bear on cases in which engineered tRNAs are expressed in cells to redirect mRNA coding (Köhrer et al. 2004) or to serve other functions, for example, as chimeras with ribozymes (Geslain et al. 2009). Would such modified tRNAs also produce small regulatory RNAs like those that have been described from normal tRNAs? All of these are intriguing questions concerning the RNA processing horizon of these new findings. 
A surprising recent finding in the tRNA biosynthesis field was that mature tRNAs traffic back into the nucleus (Hopper and Shaheen 2008). In light of the new findings reviewed here, is it possible that these returning RNAs get cleaved in the nucleus? If so, would they be exported as microRNA-like RNAs or might they perhaps perform nuclear functions? Within the nucleus, nucleoli were long ago discovered to harbor tRNAs (Sirlin 1972). Certain microRNAs have recently been localized in nucleoli (Politz et al. 2006, 2009), but these are ones that are produced via Drosha processing of typical pri-microRNA precursors. The classical evidence for tRNAs associated with nucleoli (Sirlin 1972) and more recent findings in support of this (Bertrand et al. 1998; Thompson et al. 2003) raise the question of whether tRNAs are cleaved into microRNA-like RNAs in the nucleolus.

Another question is how the coding functions of a tRNA are balanced with its role as a precursor of small regulatory RNAs. For those of the latter that are derived from the $5^{\prime}$ or $3^{\prime}$ ends of pre-tRNAs, clearly their precursors do not ever function in coding. But for those that are derived from mature tRNAs, does this occur only after they have been aminoacylated in one or more rounds of translation? If so, what then determines their fate as precursors of small regulatory RNAs? Alternatively, if there are special subsets of tRNAs that are never subject to aminoacylation, but are sent directly into the small regulatory RNA production pathway (seemingly the less likely of the two possibilities), how and where in the cell does this bifurcation take place? It is of interest to note that in $S$. pombe there is a mechanism that prevents tRNA (and ribosomal RNAs) from entering into the siRNA biogenesis pathway (Buhler et al. 2008). It is conceivable that such a mechanism operates in higher eukaryotes as well as under some conditions and perhaps happens to be relaxed or absent in the cultured cell lines in which these recent deep-sequencing studies were carried out. Alternatively, the $S$. pombe mechanism may have been selected against during the evolution of higher eukaryotes, due to a need to maximize all possible sources of small regulatory RNAs to serve the greater complexity of mRNA targets and build the necessarily more extensive RNA-mediated translational regulatory networks. As small RNA deep-sequencing studies continue in various eukaryotic organisms, at least the broad phyletic distribution of tRNA-derived small regulatory RNAs will begin to come into view.

\section{Are there other "unconventional" pre-microRNAs?}

If tRNAs produce regulatory RNAs, might other small RNAs do so as well? Recent studies have pointed to microRNA-like RNAs derived from certain of the small nucleolar RNAs (Ender et al. 2008; Kawaji et al. 2008; Scott et al. 2009; Taft et al. 2009). Moreover, the 88- or 98-nt "vault" RNAs, which are components of a ribonucleoprotein machine involved in multidrug resistance, have recently been demonstrated to produce a group of $\sim 23$-nt RNAs (Persson et al. 2009).
Although this processing step is Dicer independent, these vault RNA-derived RNAs include one that is Argonaute bound and mediates mRNA cleavage and, hence, is a microRNA by those criteria. One wonders how many other small RNAs beyond tRNAs, snoRNAs, and vault RNAs may be cut into regulatory RNAs in a pathway or pathways distinct from the canonical Drosha processing of pol II pri-miRNAs to premiRNAs and subsequent Dicer-mediated production of mature microRNAs. Perhaps pol III transcripts other than tRNAs and vault RNA are cleaved to produce regulatory RNAs? The candidates would include the small Ro RNAs (Hendrick et al. 1981), the signal recognition particle RNA (Walter and Blobel 1982), U6 spliceosomal RNA (Kunkel et al. 1986), the RNA components of RNase P and RNase MRP (Chang and Clayton 1989; Baer et al. 1990), as well as the large number of pol III transcripts produced by retrotransposons. These possibilities are just guesses at present, but continued deep sequencing studies will likely provide the answers soon enough. Meanwhile, and notwithstanding the diversity of the RNA processing pipeline that may be ultimately revealed, the recent evidence for tRNA-derived regulatory RNAs reviewed here alerts us to possibilities not previously pondered as we move along the "new RNA world," a seemingly endless frontier in our time.

\section{Transfer RNA evolution re-examined}

Transfer RNAs appear to have evolved not only as anticodons but also from short, minihelix-like ensembles that determine aminoacylation specificity (McClain 1993; Schimmel and Ribas de Pouplana 1995). These dual, cooperating motifs that determine aminoacylation specificity consist of the very $5^{\prime}$ and $3^{\prime}$ regions of tRNAs that are so emphatically represented in the deep-sequencing studies reviewed here. This surely cannot be ignored. The fact that these tRNAderived regulatory RNAs tend less often to be derived from the central regions might reflect the fact that the anticodons are configured optimally for base pairing, and would thus likely bind codons all throughout an mRNA. This suggests that it is the inclusion of either a $5^{\prime}$ or $3^{\prime}$ end of tRNA that was selected for in the evolution of these newly described RNAs and that the anticodon regions simply had no selective advantage for these mRNA regulatory functions (as opposed to coding) on which selection was operating. One thus suspects that there was nested coevolution of four entitiesthe anticodons, the aminoacylation code (residing in the $5^{\prime}$ end and the 3' acceptor stem), the aminoacyl-tRNA synthetases, and the domains producing small regulatory RNAs. The alternative possibility, viz., that the latter function arose later as what evolutionary nomenclature calls an exaptation, seems less likely. What remains then is the question of what selective pressures were brought upon the other regions of tRNAs and whether or not they are more than mere evolutionary "spandrels." In this context it is noteworthy that some of the RNAs described by Lee et al. (2009) that 
come from the $5^{\prime}$ ends of mature tRNAs included the D stem-loop.

Finally, the $5^{\prime}$ and $3^{\prime}$ elements of the investigated tRNA minihelices that have given rise to the aminoacylation specifity code are, of course, base paired in their parental full-length tRNAs. This inescapably means that some pairs of the reported tRNA-derived RNAs are complementary. Might their concentrations in the cell be sufficiently high to lead to base pairing? If so, then these duplexes of tRNA-derived RNAs could possibly be substrates for yet further cleavage (by Dicer or other RNase III activities), or even for replication by RNA-dependent RNA polymerases and the production of secondary RNAs as in RNA interference. Are such possibilities among the new things under the sun awaiting discovery in the regulatory RNA field?

\section{ACKNOWLEDGMENTS}

I am grateful to Paul Schimmel (The Scripps Research Institute) for encouragement on an early draft and for reinforcing the fact that these RNAs are mostly derived from tRNA regions that constitute the aminoacylation specificity code. I thank Victor Ambros (University of Massachusetts Medical School), Andrew Fire (Stanford University School of Medicine), and Phillip Sharp (M.I.T.) for critically commenting on an initial draft.

\section{IN REMEMBRANCE}

The discoverers of transfer RNA, Mahlon Hoagland (1920-2009) and Paul Zamecnik (1912-2009), were close colleagues of the investigator, who knows how keenly interested they would have been in these new findings. They almost lived to see them.

\section{REFERENCES}

Baer M, Nilsen TW, Costigan C, Altman S. 1990. Structure and transcription of a human gene for H1 RNA, the RNA component of human RNase P. Nucleic Acids Res 18: 97-103.

Babiarz JE, Ruby JG, Wang Y, Bartel DP, Blelloch R. 2008. Mouse ES cells express endogenous shRNAs, siRNAs, and other microprocessor-independent, Dicer-dependent small RNAs. Genes Dev 22: $2773-2785$.

Bertrand E, Houser-Scott F, Kendall A, Singer RH, Engelke DR. 1998. Nucleolar localization of early tRNA processing. Genes Dev 12: 1263-1264.

Buhler M, Spies N, Bartel DP, Moazed D. 2008. TRAMP-mediated RNA surveillance prevents spurious entry of RNAs into the Schizosaccharomyces pombe siRNA pathway. Nat Struct Mol Biol 15: 1015-1023.

Calabrese JM, Seila AC, Yoo GW, Sharp PA. 2007. RNA sequence analysis defines Dicer's role in mouse embryonic stem cells. Proc Natl Acad Sci 104: 18097-18102.

Chang DD, Clayton DA. 1989. Mouse RNase MRP RNA is encoded by a nuclear gene and contains a decamer sequence complementary to a conserved region of mitochondrial RNA substrate. Cell 56: 131-136.

Cole C, Sobala A, Lu C, Thatcher SR, Bowman A, Brown JWS, Green PJ, Barton GJ, Hutvagner G. 2009. Filtering of deep sequencing data reveals the existence of abundant Dicer-dependent small RNAs derived from tRNAs. RNA 15: 2147-2160.

Ender C, Krek A, Freidlander MR, Beitzinger M, Weinmann L, Chen W, Pfeffer S, Rajewsky N, Meister G. 2008. A human snoRNA with microRNA-like functions. Mol Cell 32: 519-528.

Geslain R, Cubells L, Bori-Sanz T, Álvarez-Medina R, Rossell D, Marti E, de Pouplana LR. 2009. Chimeric tRNAs as tools to induce proteome damage and identify components of stress responses. Nucleic Acids Res 38: e30. doi: 10.1093/nar/gkp1083.

Ghildiyal M, Zamore PD. 2009. Small silencing RNAs: An expanding universe. Nat Rev Genet 10: 94-108.

Haussecker D, Huang Y, Lau A, Parameswaran P, Fire AZ, Kay MA. 2010. Human tRNA-derived small RNAs in the global regulation of RNA silencing. RNA 16: 673-695.

Hendrick JP, Wolin SL, Rinke J, Lerner MR, Steitz JA. 1981. Ro small cytoplasmic ribonucleoproteins are a subclass of La ribonucleoproteins: Further characterization of the Ro and La small ribonucleoproteins from uninfected mammalian cells. Mol Cell Biol 1: 1138-1149.

Hoagland M. 2004. Enter transfer RNA. Nature 431: 249. doi: 10.1038/ $431249 \mathrm{a}$

Hopper AK, Shaheen HH. 2008. A decade of surprises for tRNA nuclear-cytoplamic dynamics. Trends Cell Biol 18: 98-104.

Hopper AK, Pai DA, Engelke DR. 2010. Cellular dynamics of tRNAs and their genes. FEBS Lett 584: 310-317.

Kawaji H, Nakamura M, Takahashi Y, Sandelin A, Katayama S, Fukuda S, Daub CO, Kai C, Kawai J, Yasuda J, et al. 2008. Hidden layers of human small RNAs. BMC Genomics 9: 157. doi: 10.1186/ 1471-2164-9-157.

Köhrer C, Sullivan EL, RajBhandary UL. 2004. Complete set of orthogonal $21^{\text {st }}$ aminoacyl-tRNA synthetase-amber, ochre and opal suppressor tRNA pairs: Concomitant suppression of three different termination codons in an mRNA in mammalian cells. Nucleic Acids Res 32: 6200-6211.

Kunkel GR, Maser RL, Calvet JP, Pederson T. 1986. U6 small nuclear RNA is transcribed by RNA polymerase III. Proc Natl Acad Sci 83: 8575-8579.

Lee SR, Collins K. 2005. Starvation-induced cleavage of the tRNA anticodon loop in Tetrahymena thermophila. J Biol Chem 280: 42744-42749.

Lee RC, Feinbaum RL, Ambros V. 1993. The C. elegans heterochronic gene lin- 4 encodes small RNAs with antisense complementarity to lin-14. Cell 75: 843-854.

Lee YS, Shibata Y, Malhotra A, Dutta A. 2009. A novel class of small RNAs: tRNA-derived RNA fragments (tRFs). Genes Dev 23: 2639 2649.

McClain WH. 1993. Transfer RNA identity. FASEB J 7: 72-78.

Persson E, Kvist A, Vallon-Christersson J, Medstrand P, Borg ^, Rovira C. 2009. The non-coding RNA of the multidrug resistancelinked vault particle encodes multiple regulatory small RNAs. Nat Cell Biol 11: 1268-1271.

Politz JCR, Zhang F, Pederson T. 2006. MicroRNA 206 localizes with ribosome-rich regions in both the nucleolus and cytoplasm of rat myogenic cells. Proc Natl Acad Sci 103: 18957-18962.

Politz JCR, Hogan E, Pederson T. 2009. MicroRNAs with a nucleolar location. RNA 15: 1705-1715.

Schimmel P, Ribas de Pouplana L. 1995. Transfer RNA: From minihelix to genetic code. Cell 81: $983-986$.

Scott MS, Avolio F, Ono M, Lamod AI, Barton GJ. 2009. Human miRNA precursors with box H/ACA features. PLoS Comput Biol 5: 1-13.

Sirlin JL. 1972. Biology of RNA. pp. 315-316, Academic Press, New York.

Taft RJ, Glazov EA, Lassmann T, Hayashizaki Y, Carninci P, Mattick JS. 2009. Small RNAs derived from snoRNAs. RNA 15: 1233-1240.

Thompson M, Hoeusler RA, Good PD, Engelke DR. 2003. Nucleolar clustering of dispersed tRNA genes. Science 302: 1399-1401.

Walter P, Blobel G. 1982. Signal recognition particle contains a $7 S$ RNA essential for protein translocation across the endoplasmic reticulum. Nature 299: 691-698.

Wightman B, Ha I, Ruvkun G. 1993. Posttranscriptional regulation of the heterochronic gene lin- 14 by lin- 4 mediates temporal pattern formation in C. elegans. Cell 75: 855-862.

Yeung ML, Bennasser Y, Watashi K, Le S-Y, Houzet L, Jeang K-T. 2009. Pyrosequencing of small noncoding RNAs in HIV-1 infected cells: Evidence for the processing of a viral-cellular doublestranded RNA hybrid. Nucleic Acids Res 19: 6575-6586.

Zamboni MA, Scarabino DA, Tocchini-Valentini GP. 2009. Splicing of mRNA mediated by tRNA sequences in mouse cells. RNA 15: $2122-2128$. 

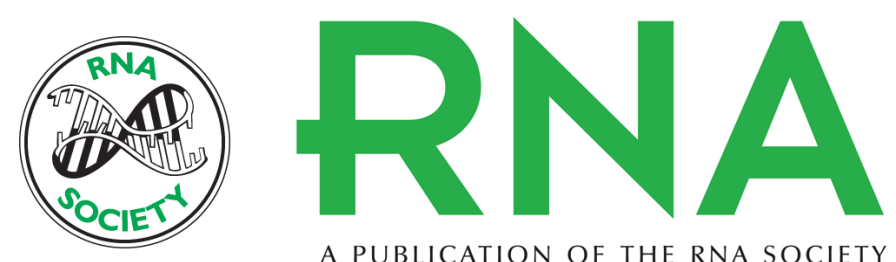

A PUBLICATION OF THE RNA SOCIETY

\section{Regulatory RNAs derived from transfer RNA?}

\section{Thoru Pederson}

RNA 2010 16: 1865-1869 originally published online August 18, 2010

Access the most recent version at doi:10.1261/rna.2266510

\section{References This article cites 33 articles, 13 of which can be accessed free at: http://rnajournal.cshlp.org/content/16/10/1865.full.html\#ref-list-1}

\section{License}

Email Alerting Receive free email alerts when new articles cite this article - sign up in the box at the Service top right corner of the article or click here.

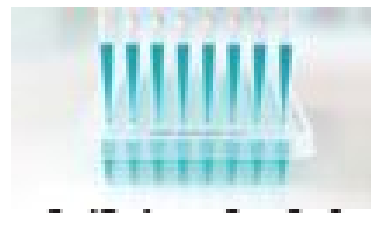

\section{Providing Precise Solutions for} your research.

To subscribe to RNA go to:

http://rnajournal.cshlp.org/subscriptions 